\title{
Microwave absorption by an array of carbon nanotubes: A phenomenological model
}

\author{
Z. Ye, W. D. Deering, A. Krokhin, and J. A. Roberts \\ Department of Physics, University of North Texas, Denton, Texas 76203-5370, USA \\ (Received 1 March 2006; revised manuscript received 19 May 2006; published 29 August 2006)
}

\begin{abstract}
A simple model to explain microwave-induced heating of carbon nanotubes (CNTs) through transformation of electromagnetic energy into mechanical vibrations is proposed and analyzed. The model provides a way to understand recent observations of heating of CNTs exposed to microwaves in the range of $2-20 \mathrm{GHz}$. It is shown that transverse vibrations of CNTs during microwave irradiation can be associated with parametric resonance, as occurs in the analysis of acoustic experiments on forced longitudinal vibrations of a stretched elastic string. For carbon nanotubes [single wall nanotube (SWNT), double wall nanotube (DWNT), multiwall nanotube (MWNT), ropes, and strands] the resonant parameters are shown to be located in a region of instability of the Mathieu's equation. Wave equations with cubic nonlinearity were used to qualitatively describe the effects of phonon-phonon interactions and energy transfer from microwaves to CNTs at a rate much exceeding the traditional Joule heating via electron-phonon interaction.
\end{abstract}

DOI: 10.1103/PhysRevB.74.075425

\section{INTRODUCTION}

The responses of carbon nanotubes to electromagnetic radiation over a broad range of frequencies have been the topic of many recent investigations. ${ }^{1-8}$ Visible laser-induced spectrofluorences ${ }^{1,2}$ and Raman spectra, ${ }^{3}$ ultrafast laser pulseinduced structure change, ${ }^{4}$ and irradiation from a lower frequency microwave source with emissions detected at higher, infrared, and visible, frequencies ${ }^{5-8}$ have been reported. A phenomenological model to describe the interaction of microwaves with carbon nanotubes (CNTs) is proposed and used to explain a set of experimental observations.

In 2004, Roberts et al. $^{5}$ reported specific microwave absorption in the frequency range $6 \mathrm{GHz}$ to $20 \mathrm{GHz}$ by CNTs blended in polycarbonate. Imholt et al. ${ }^{6}$ and Wadhawan et al. ${ }^{7}$ reported that CNTs under vacuum (less than $10^{-5}$ Torr) irradiated with a microwave field of $300 \mathrm{~W}$ produced a visibly bright plasma accompanied by rapid degassing of the CNTs. Naab et al. ${ }^{8}$ repeated the experiments of Refs. 6 and 7 under the same microwave field conditions, using predegassed CNTs. The authors in Ref. 8 reported that the unpurified samples were uniformly heated, but purified samples were not uniformly heated. Wadhawan et al. ${ }^{7}$ compared the distribution of the residual metal in the CNT samples before and after irradiation and proposed that the heating of the sample is the result of magnetic resonance of the residual metal in the microwave field. Naab et al., ${ }^{8}$ using a particleinduced $\mathrm{x}$-ray emission (PIXE) experiment, showed a lack of correlation between the temperature of CNTs and the amount of residual metals. Walton et al. ${ }^{9}$ reported that nanosized ferromagnetic grains show temperature increases under microwave radiation for higher frequencies than $2.45 \mathrm{GHz}$, as used in Refs. 5-8.

This paper proposed to answer the questions: What is the role of residual metals in the heating process? Is there any correlation between the microwave heating of the CNTs and plasma excitation? Why are unpurified CNTs uniformly heated through the Joule process but purified CNTs are nonuniformly "over" heated?

Two types of mechanical response of CNTs to an alternating electric field were studied. In 1999, Poncharal et al. ${ }^{10}$ observed deflections of cantilevered multiwall nanotubes
(MWNTs) with resonant frequencies ranging from $\mathrm{KHz}$ to MHz. The CNT was set in vibrational motion with one end held fixed and the other free. In 2000, Reulet et al. ${ }^{11}$ measured acoustoelectric effects of single wall nanotube (SWNT) ropes of $2 \mu \mathrm{m}$ lengths when subjected to $1 \mathrm{~K}$ and showed for transverse resonant frequencies ranging from $100 \mathrm{MHz}$ to $10 \mathrm{GHz}$, electron-phonon interaction is up to 1000 times greater than the values at other frequencies.

Fundamental to the work of Reulet et al. ${ }^{11}$ is the charge depletion between a CNT and metal contact because of the difference of work functions between the two kinds of materials, contacts, and sample, which produce nonuniform charge distribution. In 1984, Ekardt ${ }^{12}$ systematically studied work functions of metal clusters which varied with the number of atoms in the clusters and showed that as long as the number of atoms in a cluster is over 100, the value of the work function of the cluster approaches the value of the bulk metal.

Reulet's mechanism is insufficient to explain the microwave heating of CNTs in Refs. 6-8. The reasons that led us to develop a new model were that in Ref. 8, microwave heating of purified CNTs exhibited two extreme phenomena: CNTs were either heated to "super" high temperature or not heated, with no intermediate situation. This phenomenon could not be understood within Reulet's mechanism, where a CNT rope was treated as a transversely excited charged elastic rod with both ends fixed.

Polarization of CNTs under both static and alternating electric fields has been studied in Refs. 13-16. Both theoretical and experimental analyses showed that under an external electric field, a CNT would be polarized along its axial direction. Under a static electric field, polarization of a CNT results in a mechanical extension of the CNT along its axis. ${ }^{15}$ A similar extension occurs in a microwave field, which can be considered as quasistatic for tubes of lengths $\sim 1 \mu \mathrm{m}$. From fundamental concepts, it is estimated that a CNT of $L=1 \mu \mathrm{m}, d=1 \mathrm{~nm}$, and Young's modulus of $1 \mathrm{TPa}$ under an electric field of $10^{4} \mathrm{~V} / \mathrm{cm}$ intensity and a total number of $10^{2}$ free electrons depleted from any residual metal develops a strain of order $10^{-4}$. In addition, the aspect ratio, length divided by diameter, ${ }^{17}$ must be considered. For an aspect ratio less than 10, a cylindrical shell model should be taken; if the 
aspect ratio is from 10 to 50 , a rigid rod model should be used; if the ratio is larger than 500, a wire or string model is suitable. We considered the aspect ratio to be 1000 and assumed a straight, classical, extensible, and flexible string with instantaneous mechanical response to the microwave field parallel to the string. The resulting tension is assumed to be uniform along the string, with the ends free with atomic degrees of freedom neglected. The CNT motions that open an energy input window for microwaves are considered while neglecting the motions that cause heating. (Many authors have systematically studied electron-phonon interaction of CNTs. See Ref. 18 and references contained therein.) Here we assume that microwave heating of the CNTs begins with resonant excitation of transverse wave motion followed by electron-phonon and phonon-phonon interactions. Periodic variation of the tension of the string leads to transverse parametric resonance and unstable motion. Using the third-order longitudinal-transverse, coupled, nonlinear wave equations given by Morse and Ingard, ${ }^{19}$ the resonant solutions are shown to be pseudounstable. The displacements are solutions of a nonlinear Mathieu equation derived from the third-order wave equations constrained to two-dimensional wave motion. Initial conditions of the nonlinear Mathieu's equation are considered given (referred to as a "seed" by Rowland ${ }^{20}$ ) and initiated by thermal excitations. (Experiments 6-8 were done at room temperature in the work cited in Ref. 20.)

\section{THE MODEL}

A CNT interacting with a spatially uniform electric field at a microwave frequency $\omega_{E}$ pointing along the tube axis induces polarization that responds instantly to the field. This polarization causes an antenna effect in illuminated arrays of $\mathrm{CNTs}^{21}$ that produces forces in opposite directions on the ends of the tube, giving rise to a mechanical frequency $2 \omega_{E}$.

If the equilibrium length $L_{0}$ of the stretched CNT is set at $L_{r}+\left(L_{M}-L_{r}\right) / 2$, for the frequency of the driving force $2 \omega_{E}$, the CNTs under a microwave field behaves almost the same as that of an elastic string in Melde's acoustic experiment. ${ }^{22-25}$ Herein, both ends are free to move transversely, whereas in the acoustic experiment both ends are fixed. Rowland's analysis of the Melde experiment to the microwave-driven CNT problem ${ }^{20}$ is adopted in this work.

If the $x$ axis is chosen to lie along the resting CNT, a point on the string initially at $(x, 0,0)$, is found at time $t>0$ to be at the point

$$
\mathbf{r}(t)=\{x+u(x, t), v(x, t), w(x, t)\} .
$$

At the free ends, the boundary conditions are

$$
\begin{gathered}
u(0, t)=u_{0} \cos \left(2 \omega_{E} t\right), \\
u\left(L_{0}, t\right)=-u_{0} \cos \left(2 \omega_{E} t\right),
\end{gathered}
$$

and

$$
v_{x}(0, t)=v_{x}\left(L_{0}, t\right)=w_{x}(0, t)=w_{x}\left(L_{0}, t\right)=0,
$$

where $u_{0}=\left(L_{M}-L_{r}\right) / 2$ is a function of the microwave power and is dependent on the electronic energy structure, ${ }^{14} v_{x}$ $=\partial v / \partial x$, etc. Boundary conditions, Eqs. (2) and (3), cause the tension $\tau$ in the tube to change in time according to Hooke's law,

$$
\tau=S Y \frac{\left[L_{0}-2 u_{0} \cos \left(2 \omega_{E} t\right)-L_{r}\right]}{L_{r}}=\tau_{0}\left[1-b \cos \left(2 \omega_{E} t\right)\right],
$$

with

$$
\tau_{0}=S Y \frac{\left(L_{0}-L_{r}\right)}{L_{r}}
$$

and $b=2 u_{0} /\left(L_{0}-L_{r}\right)=2$, from the definitions of $u_{0}$ and $L_{0} ; S$ is the cross-sectional area of the CNT (or nanorope, nanostrand), $Y$ is Young's modulus, and $\tau_{0}$ is the tension of the nanotube in equilibrium. If $\varepsilon$ is the equilibrium strain, a $(17,17)$-type SWNT with a length of $1 \mu \mathrm{m}$ and strain of $10^{-4}$ $\left(L_{0}-L_{r}=1 \AA\right)$ with $Y=1 \mathrm{TPa}$, the fundamental transverse mode frequency is approximately $2.1 \mathrm{GHz}$, which is near the microwave frequency of $2.45 \mathrm{GHz}$ used in the experiments.

\section{A. The linear model}

The equation for the motion of the nanotube for timedependent tension using Eq. (5) gives

$$
\frac{\partial^{2} v}{\partial t^{2}}-c_{T}^{2}\left[1-b \cos \left(2 \omega_{E} t\right)\right] \frac{\partial^{2} v}{\partial x^{2}}=0,
$$

where $c_{T}=\sqrt{\tau_{0} / \rho_{0}}$ is the speed of transverse waves. Expanding the solutions over odd harmonics with suitable replacements gives the Mathieu equation for $v_{n}(t)$,

$$
\frac{d^{2} v_{n}}{d T^{2}}+[\alpha-\beta \cos (T)] v_{n}=0,
$$

where $\alpha=\beta / 2=\omega_{n}^{2} /\left(4 \omega_{E}^{2}\right), \omega_{n}=c_{T} k_{n}, T=2 \omega_{E} t$; the integer $n$ is odd to keep the center of mass stationary. A resonance occurs for $\omega_{n} \approx \Omega / 2$, where $\Omega$ is the frequency of the driving force ${ }^{26}$ In our case $\Omega=2 \omega_{E}$ and resonance occurs for $\omega_{n}$ $\approx \omega_{E}=2.45 \mathrm{GHz}$ with $n=1$, so that $\alpha=\beta / 2 \approx 1 / 4$. The solutions of Eq. (8) are stable or unstable depending on the values of $\alpha$ and $\beta$, as shown in the $(\alpha-\beta)$ parameter space in Fig. 1. The boundaries between stable and unstable regions near the values $\alpha=1 / 4, \beta=0$ are given by $\alpha \approx 1 / 4 \pm \beta / 2$ $-\beta^{2} / 8^{20,27,28}$ It is seen that the point $\alpha=1 / 4, \beta=1 / 2$ lies in an unstable region and the general solution has the form

$$
v_{n}(t)=c_{1} e^{\mu T} q_{1}(T)+c_{2} e^{-\mu T} q_{2}(T),
$$

where $\mu$ is a real number depending on $\alpha$ and $\beta$, and $q_{1}$ and $q_{2}$ are periodic functions. The exponentially growing solution in Eq. (9) holds for continuous ranges of values of $\alpha$ and $\beta$ (Fig. 1). For parametric resonance, the result is either unlimited growth for damping $\kappa$ less than a critical value $\kappa$ $<2 \mu$, or overdamped transverse motion for damping larger than the critical value. If the interaction between the CNT and its surroundings is included through the addition in Eq. (8) of a linear damping term of the form $\kappa d \nu / d T$ with the transformation $\nu_{n}=\exp (-\kappa T / 2) \psi(T)$ made to eliminate the first derivative term, $\psi$ satisfies another Mathieu equation, of the form 


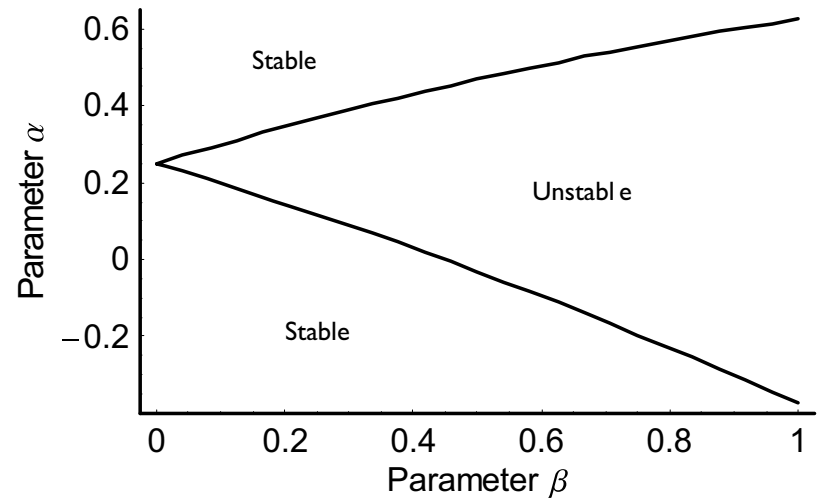

FIG. 1. Near $\beta=0$, the parameter space is divided into regions corresponding to stable and unstable solutions of the Mathieu equation. The regions are separated by the lines $\alpha=1 / 4 \pm \beta / 2-\beta^{2} / 8$. Melde's experiment corresponds to $\alpha=\beta / 2=1 / 4$.

$$
\frac{d^{2} \psi}{d T^{2}}+[\eta-\beta \cos (T)] \psi=0,
$$

where $\eta=\alpha-\kappa^{2} / 4$. The solution for $\nu_{n}$ is

$$
v_{n}=c_{1} e^{(\mu-\kappa / 2) T} q_{1}(T)+c_{2} e^{-(\mu+\kappa / 2) T} q_{2}(T) .
$$

The case $\kappa>2 \mu$ yields no lasting transverse motion. For $\kappa$ $<2 \mu$, transverse motion persists and is unbounded. Any motion of interest remains unstable in the presence of damping. Note that the boundaries between stable and unstable regions in parameter space near $\left(\alpha-\kappa^{2} / 4\right)=1 / 4$ and $\beta<1$ are given by $\alpha=1 / 4 \pm\left(\beta^{2}-\kappa^{2}\right)^{1 / 2} / 2,{ }^{25}$ or

$$
\frac{\omega}{\omega_{E}}=\sqrt{1 \pm 2 \sqrt{\beta^{2}-\kappa^{2}}}
$$

\section{B. The nonlinear model}

It can be seen that the linear solution is not sufficient for long times, as the amplitude of the transverse wave grows exponentially and is not limited by damping, so the linear wave equations must be modified to include nonlinear terms. Following Morse and Ingard, ${ }^{19}$ including nonlinear effects that couple longitudinal and transverse displacements leads to the set of coupled equations with cubic nonlinearity:

$$
\begin{gathered}
\frac{\partial^{2} u}{\partial t^{2}}-c_{L}^{2} \frac{\partial^{2} u}{\partial x^{2}}=\frac{1}{2}\left(c_{L}^{2}-c_{T}^{2}\right) \frac{\partial}{\partial x}\left[\left(v_{x}^{2}+w_{x}^{2}\right)\left(1-2 u_{x}\right)\right], \\
\frac{\partial^{2} v}{\partial t^{2}}-c_{T}{ }^{2} \frac{\partial^{2} v}{\partial x^{2}}=\frac{1}{2}\left(c_{L}^{2}-c_{T}^{2}\right) \frac{\partial}{\partial x}\left[v_{x}\left(v_{x}^{2}+w_{x}^{2}\right)+2 u_{x} v_{x}\left(1-u_{x}\right)\right]
\end{gathered}
$$

where $u_{x} \equiv \partial u / \partial x$, etc. An equation for $w$ comes from Eq. (15) by interchanging $v$ and $w$; however, we consider only planar motion $(w \equiv 0)$ and take $u \ll 1$. Here $c_{L}=2.0$ $\times 10^{6} \mathrm{~cm} / \mathrm{s}$ (Ref. 29) is the speed of longitudinal waves. The speed of transverse waves $c_{T}$ is less than one-tenth of $c_{L}$, which is used to simplify Eq. (15) to get an approximate connection between $u$ and $v$. Keeping only the dominant terms in Eq. (15) after dividing by $c_{L}$ and letting $c_{L} \rightarrow \infty$ the equation for $u$ above then reduces to

$$
\frac{\partial^{2} u}{\partial x^{2}}=-\frac{1}{2} \frac{\partial}{\partial x}\left(v_{x}^{2}\right)
$$

The last equation can be integrated over $x$, subject to boundary conditions Eqs. (2) and (3), to get

$$
\begin{aligned}
\frac{\partial^{2} v}{\partial t^{2}} & -c_{T}^{2} \frac{\partial^{2} v}{\partial x^{2}} \\
& =\frac{1}{2} \frac{\left(c_{L}^{2}-c_{T}^{2}\right)}{L_{0}}\left(-\frac{4 u_{0} \cos \left(2 \omega_{E} t\right)}{L_{0}}+\frac{1}{L_{0}} \int_{0}^{L_{0}} v_{x}^{2} d x\right) \frac{\partial^{2} v}{\partial x^{2}} .
\end{aligned}
$$

The coupled nonlinear Mathieu equations for $v_{n}$ can be written as follows

$$
\frac{d^{2} v_{n}}{d T^{2}}+\left[\alpha_{n}-\beta_{n} \cos (T)\right] v_{n}+\gamma_{n n} v_{n}^{3}+\sum_{j \neq n} \gamma_{n j} v_{j}^{2} v_{n}=0,
$$

where

$$
\begin{gathered}
\alpha_{n}=n^{2} \alpha_{1}, \quad \alpha_{1}=\left(\omega_{1} / 2 \omega_{E}\right)^{2}, \quad \beta_{n}=2 n^{2} \alpha_{1}, \\
\gamma_{n n}=(n \pi)^{2} \alpha_{n} /(4 \varepsilon), \quad \gamma_{n j}=(j \pi)^{2} \alpha_{n} /(4 \varepsilon) .
\end{gathered}
$$

We take $\alpha_{1}=\frac{1}{4}$, solve the two equations for $n=1$ and $n=3$, and then find the Fourier amplitude spectra for $v_{1}$ and $v_{3}$. The results are plotted in Figs. 2 and 3. The abscissas are the integers $p$ in the angular frequencies $2 \pi p / t_{0}$, where $t_{0}$ is connected to the dimensionless time interval $T_{0}$, used in solving Eq. (16), by $T_{0}=2 \omega_{E} t_{0}=500$. The primary $v_{1}$ peak occurs near the physical frequency of $2 \pi(54 / 250) \omega_{E} \approx 1.36 \omega_{E}$ and the lower secondary peak near $p=28$, is near an angular frequency of $0.70 \omega_{E}$; both are near the microwave field frequency, as expected. The amplitudes for $v_{3}$ are smaller than

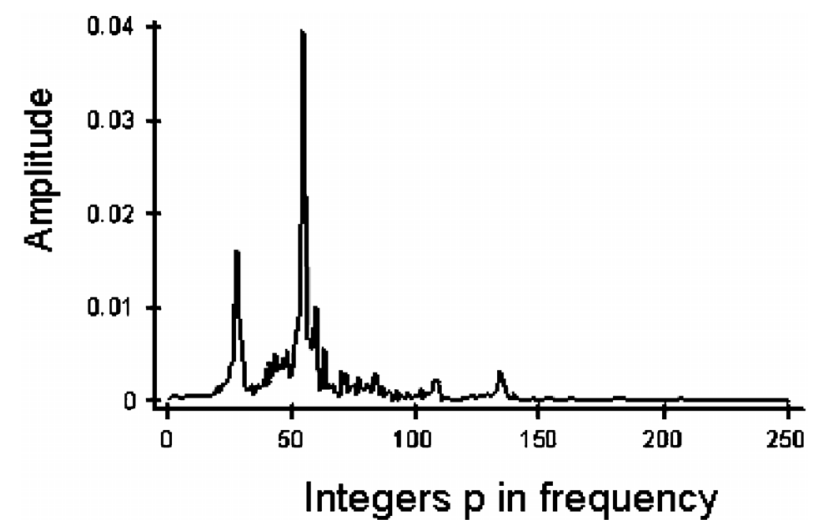

FIG. 2. The absolute values of the discrete Fourier amplitudes of the nonlinear mode amplitude $\nu_{1}(t)$ are plotted against the integers $p$ in the angular frequencies $2 \pi p / t_{0}$, with $t_{0}=250 / \omega_{E}$. The primary peak occurs near $p=54$, or near an angular frequency of $\pi p \omega_{E} / 125=1.36 \omega_{E}$, above the microwave frequency used to irradiate the CNTs. The lower secondary peak near $p=28$ is near an angular frequency of $0.70 \omega_{E}$. 


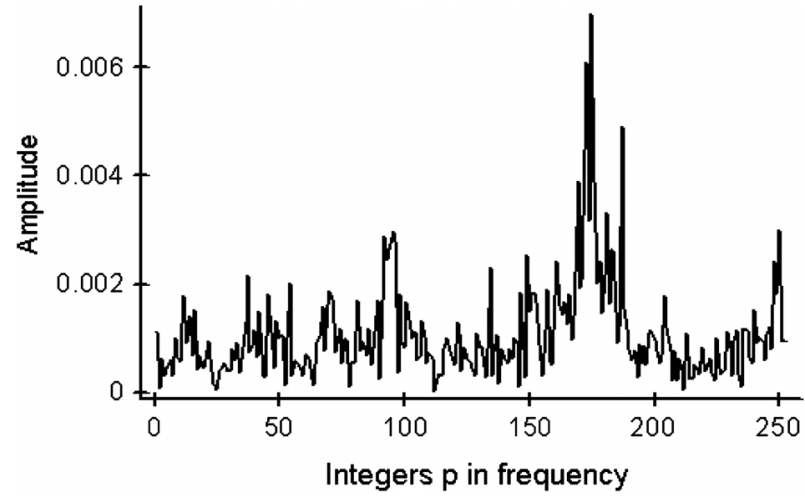

FIG. 3. The absolute values of the discrete Fourier amplitude spectrum of the nonlinear mode amplitude $\nu_{3}(t)$ is plotted versus the integers $p$ in the angular frequencies $2 \pi p / t_{0}$, with $t_{0}=250 / \omega_{E}$. The primary peak occurs near $p=174$, or near an angular frequency of $\pi p \omega_{E} / 125=4.37 \omega_{E}$, over four times the frequency used to irradiate the CNTs.

for $v_{1}$, and the spectrum has higher frequencies compared to that for $v_{1}$. The primary $v_{3}$ peak is near $2 \pi(174 / 250) \omega_{E}$ $\approx 4.37 \omega_{E}$. Coupling of the $v$ modes feeds energy from the electric field by way of the fundamental into other higher frequency modes. Figure 1 shows the stable and unstable domains of the solutions of the non-linear solutions.

\section{RELATING THE MODEL TO SOME EXPERIMENTAL OBSERVATIONS}

Now we connect the behavior of the model presented in Sec. II with the observations discussed in the Introduction. The model predicts that the behavior of a CNT under a microwave field is described by solutions of the nonlinear Mathieu equation, Eq. (16). The form of the solution is determined by reference to its $(\alpha-\beta)$ parameter space, Fig. 1. At resonance, $\beta=1 / 2$ is a constant, but $\alpha=1 / 4-\kappa^{2} / 4$ is a function of the damping from Eq. (10) and its value determines if the solution is stable or unstable. Also, Eq. (12) shows that damping causes a shift of resonant frequency, which is controlled by the amount of damping.

When $\kappa$ is large enough (e.g., $\kappa>1>\beta$ ), the CNT systems in Refs. 5-8 are overdamped and there is no steady motion. In this case, the states of the systems are somewhere below the $\alpha=0$ line in $(\alpha-\beta)$ parameter space, lying in a neighboring unstable region (not shown in Fig. 1), but having solutions with only decaying exponentials in Eq. (13) and indicating that the CNTs are not in resonance with the field.

Solutions for the pregassed, unpurified CNTs, because of the presence of impurities, were limited by the $\beta=\frac{1}{2}$ line as far as the stable region shown in the $(\alpha-\beta)$ parameter space in Fig. 1. In this region, transverse resonance with a finite amplitude holds for all the CNTs. Based on the results in Ref. 8, electron-phonon interaction is significant and, therefore, uniform Joule heating occurs in this case.

In solutions for the purified CNTs, because there is less damping from the impurities, pre-degassing enables the samples to pass the $\beta=\frac{1}{2}$ line into the unstable region of the $(\alpha-\beta)$ parameter space. Degassing of the purified CNTs in Ref. 8 results in a nonuniform density distribution of the CNTs. For the ones whose local environment allows them to take on large amplitude vibrations, they will vibrate with increasing amplitudes until nonlinear effects become involved, as in Eq. (16). In this case, besides Joule heating caused by electron-phonon interactions, we must also consider heating effects due to phonon-phonon interactions, resulting from large-amplitude nonlinear transverse motion. For the CNTs in dense and viscous local environments, the CNTs will not vibrate. As a result, in these CNTs there is neither phonon-phonon coupling nor electron-phonon interaction, and thus, no heating.

\section{CONCLUSIONS}

In this work is presented a discussion of the interaction of CNTs with microwaves in terms of the nonlinear dynamics of CNTs modeled as an elastic string. The model predicts a primitive excitation of a $\mathrm{CNT}$ at room temperature that involves all atoms in the CNT under intense microwave irradiation. Melde's experiment and its description in terms of Mathieu's equation are useful in trying to understand the dynamical response of CNTs during microwave irradiation. It is pointed out that the damping coefficient $\kappa$ plays a key role in determining the behavior of nanotubes. When the damping is too large, transverse waves cannot be excited; when $\kappa \ll \beta$ but $\kappa>0$ electron-phonon interaction (Joule heating) dominates the energy conversion process. Phononphonon interaction must be considered whenever $\kappa \rightarrow 0$. In the case reported here, resonant response of CNTs to microwaves results in two effects, an increase in electron-phonon scattering and the appearance of nonlinear effects and phonon-phonon scattering. This model also implies significant density effects, as experimentally observed in Ref. 5 and as mentioned in Sec. III. Finally, it is pointed out that during microwave irradiation, residual metals in the system appear to play the role of donors of sufficient free charge to expedite the microwave coupling.

\section{ACKNOWLEDGMENT}

Part of this research was supported by the Office of Naval Research, Contract No. ONR N00013-03-1-0880. 
${ }^{1}$ S. M. Bachilo, M. S. Strano, C. Kittrell, R. H. Hauge, R. E. Smalley, and R. B. Weisman, Science 298, 2361 (2002).

${ }^{2}$ G. N. Ostojic, S. Zaric, J. Kono, M. S. Strano, V. C. Moore, R. H. Hauge, and R. E. Smalley, Phys. Rev. Lett. 92, 117402 (2004).

${ }^{3}$ A. Thess, R. Lee, P. Nikolaev, H. Dai, P. Petit, J. Robert, C. Xu, Y. H. Lee, S. G. Kim, A. G. Rinzler, D. T. Colbert, G. E. Scuseria, D. Tománek, J. E. Fischer, and R. E. Smalley, Science 273, 483 (1996); A. M. Rao, E. Richter, S. Bandow, B. Chase, P. C. Eklund, K. A. Williams, S. Fang, K. R. Subbaswamy, M. Menon, A. Thess, R. E. Smalley, G. Dresselhaus, and M. S. Dresselhaus, ibid. 275, 187 (1997); A. Jorio, G. Dresselhaus, M. S. Dresselhaus, M. Souza, M. S. S. Dantas, and M. A. Pimenta, A. M. Rao, R. Saito, C. Liu, and H. M. Cheng, Phys. Rev. Lett. 85, 2617 (2000).

${ }^{4}$ T. Dumitrica, M. E. Garcia, H. O. Jeschke, and B. I. Yakobson, Phys. Rev. Lett. 92, 117401 (2004).

${ }^{5}$ J. A. Roberts, T. Imholt, Z. Ye, C. A. Dyke, D. W. Price, Jr., and J. M. Tour, J. Appl. Phys. 95, 4352 (2004).

${ }^{6}$ T. J. Imholt, C. A. Dyke, B. Hasslacher, J. M. Perez, D. W. Price, J. A. Roberts, J. B. Scott, A. Wadhawan, Z. Ye, and J. M. Tour, Chem. Mater. 15, 3969 (2003).

${ }^{7}$ A. Wadhawan, D. Garrett, and J. Perez, Appl. Phys. Lett. 83, 2683 (2003).

${ }^{8}$ F. Naab, M. Dhoubhadel, W. Holland, J. Duggan, J. Roberts, and F. McDaniel, in Proceedings of the 10th International Conference on Particle Induced X-ray Emission and Analytical Applications, Portoroz, Slovenia, 2004 (Wiley Interscience, Hoboken, NJ, 2005).

${ }^{9}$ D. Walton, H. Boehnel, and D. J. Dunlop, Appl. Phys. Lett. 85, 5367 (2004).

${ }^{10}$ P. Poncharal, Z. L. Wamg, D. Ugarts, and W. A. de Heer, Science 283, 1513 (1999).

${ }^{11}$ B. Reulet, A. Yu. Kasumov, M. Kociak, R. Deblock, I. I. Khodos, Yu. B. Gorbatov, V. T. Volkov, C. Journet, and H. Bouchiat, Phys. Rev. Lett. 85, 2829 (2000).

${ }^{12}$ W. Ekardt, Phys. Rev. B 29, 1558 (1984).
${ }^{13}$ L. X. Benedict, S. G. Louie, and M. L. Cohen, Phys. Rev. B 52, 8541 (1995).

${ }^{14}$ F. Torrens, Nanotechnology 15, S259 (2004).

${ }^{15}$ Y. Guo and W. Guo, J. Phys. D 36, 805 (2003).

${ }^{16}$ A. Jorio, A. G. Souza Filho, V. W. Brar, A. K. Swan, M. S. Ünlü, B. B. Goldberg, A. Righi, J. H. Hafner, C. M. Lieber, R. Saito, G. Dresselhaus, and M. S. Dresselhaus, Phys. Rev. B 65, 121402 (2002).

${ }^{17}$ M. J. Buehler, Y. Kong, and H. Gao, J. Eng. Mater. Technol. 126, 245 (2004)

${ }^{18}$ T. Hertel and G. Moos, Phys. Rev. Lett. 84, 5002 (2000).

${ }^{19}$ P. M. Morse and K. U. Ingard, Theoretical Acoustics (McGrawHill, New York, 1968).

${ }^{20}$ D. R. Rowland, Am. J. Phys. 72, 758 (2004).

${ }^{21}$ Y. Wang, K. Kempa, B. Kimball, J. B. Carlson, G. Benham, W. Z. Li, T. Kempa, J. Rybczynbski, A. Herczynski, and Z. F. Ren, Appl. Phys. Lett. 85, 2607 (2004).

${ }^{22}$ M. Y. Colby, Sound Waves and Acoustics (Henry Holt, New York, 1938).

${ }^{23}$ E. G. Richardson, Sound: A Physical Text-Book (Edward Arnold, London, 1927).

${ }^{24}$ N. W. McLachlan, Theory and Applications of Mathieu Functions (Oxford, London, 1947).

${ }^{25}$ N. W. McLachlan, Ordinary Non-Linear Differential Equations (Oxford, London, 1956).

${ }^{26}$ L. D. Landau and E. M. Lifshitz, Mechanics (Pergamon, Elmsford, NY, 1976).

${ }^{27}$ M. Abramowitz and I. E. Stegun, Handbook of Mathematical Functions (U.S. Department of Commerce, Washington, DC, 1972).

${ }^{28}$ D. W. Jordan and P. Smith, Nonlinear Ordinary Differential Equations, 3rd ed. (Oxford University Press, Inc., New York, 1999), Chap. 9.

${ }^{29}$ A. Mizel, L. X. Benedict, M. L. Cohen, S. G. Louie, A. Zettl, N. K. Budraa, and W. P. Beyermann, Phys. Rev. B 60, 3264 (1999). 